Volume 10. No.2, February 2022

International Journal of Emerging Trends in Engineering Research

Available Online at http://www.warse.org/IJETER/static/pdf/file/ijeter051022022.pdf

https://doi.org/10.30534/ijeter/2022/051022022

\title{
Use of Smart Wearable Devices for the Acquisition and Subsequent Analysis of the Stress Level of a University Professor
}

\author{
Luz Moreno ${ }^{1}$, Cesar Peña ${ }^{2}$ and Mauricio Rojas ${ }^{3}$ \\ ${ }^{1}$ INGPRO-GES and GICE groups, University of Pamplona, Colombia, luz.moreno@ unipamplona.edu.co \\ ${ }^{2}$ Automation and Control group, University of Pamplona, Colombia, cesarapc@unipamplona.edu.co \\ ${ }^{3}$ CICOM group, University of Pamplona, Colombia, mrojas@ unipamplona.edu.co
}

Received Date : December 30, 2021 Accepted Date : January 26, 2022 Published Date : February 07, 2022

\begin{abstract}
This paper presents the use of a wearable device as a low-cost technological tool for estimating the stress level of a university professor. The wearable device has infrared sensors that, when in contact with the skin, allow it to know the variations in the user's heart rate and indirectly estimate stress levels through an endorsed model. An experimental methodology with a non-probabilistic convenience sample was proposed. The period of experimental evaluation was one year, 24 hours a day. A classification of the levels of stress that the university professor manages was made: relaxed, normal, medium and high. This made it possible to show the percentage of time that he held in each of these categories. Within the quantitative analysis, a differentiation was made between working days, weekends and holidays. As significant results, it was found that the working day with the highest stress values was Wednesday with a value of $\% 59.78$ and, in a non-presumptive way, Saturday was detected, despite not being a working day, the university professor reached significant levels of stress. It was possible to show that the use of low-cost wearable devices allows an estimation of the repercussions at the stress level of workloads, for which it can serve as a tool for planning and scheduling tasks.
\end{abstract}

Key words : Analysis, stress, wearable device, workload.

\section{INTRODUCTION}

Today. The work demands and lifestyles of people involve the development of a large number of tasks in a short time. It is very important to keep a calm mind to efficiently carry out daily activities. Good stress management brings productivity benefits and reduces the risk of illness.

There are various techniques that allow measuring the stress of a person; the most popular correspond to some neuro-psychological tests [1]-[4]. At present, thanks to the rise of neurosciences, new techniques have been generated, among which the following stand out: the use of the magneto-encephalogram (MEG) [5], the processing of functional and anatomical magnetic resonance images (fMRI, MRI) [6], [7] and the analysis of encephalographic signals. The first three techniques usually involve high costs and the last technique has given rise to the famous brain-computer interfaces (BCI) [8], [9]. BCIs are non-invasive devices that allow the capture and evaluation of brain electrical activity in real time [10]. There are a wide variety of BCIs on the market, most of which offer great advantages, however they have some limitations such as limited autonomous operation due to batteries and sometimes the electrodes can cause discomfort to users. These characteristics mean that users do not usually use BCIs for long records.

In this research, it is proposed to carry out a study in which a record of the stress levels of a university teacher is made for a considerable period of time, in order to analyze the days of greatest and least stress and generate possible recommendations on the distribution of academic activities throughout the week. It should be considered that this study is carried out individually, taking into account that teachers tend to have a different distribution of academic, administrative and research loads. It is intended to serve as a model as an example of the application of physics for the development of technological tools and their use in the area of education.

\section{MATERIALS AND METHODS}

For the development of the project, a smartwatch was selected that had optical sensors at the bottom. These sensors have the ability to appreciate blood flow allowing them to estimate heart rate. This is done, since there is a relationship between a person's stress level and their heart rate [11], [12]. This relationship allowed the development of a stress level estimation technique based on pre-established stress levels and heart rate measurement [13], [14], this technique has been certified by the Institute of Psychology and the Chinese 
Academy of Sciences as lo reports the mobile device manufacturer Huawei.

Figure 1 shows the smartwatch, whose reference is the GT2, manufactured by Huawei. It is a device with a diameter of 46 millimeters and an approximate weight of 41 grams, which makes it very comfortable for the user. The back of the device has two light-emitting diodes, which when activated illuminate the user's skin, allowing the presence of blood in the area to be distinguished by means of two photoreceptors called optical sensors. Changes in the presence of blood in the area allow estimating the heart rate. Figure also shows the arm of a user with the smartwatch and how the sensors illuminate the skin.

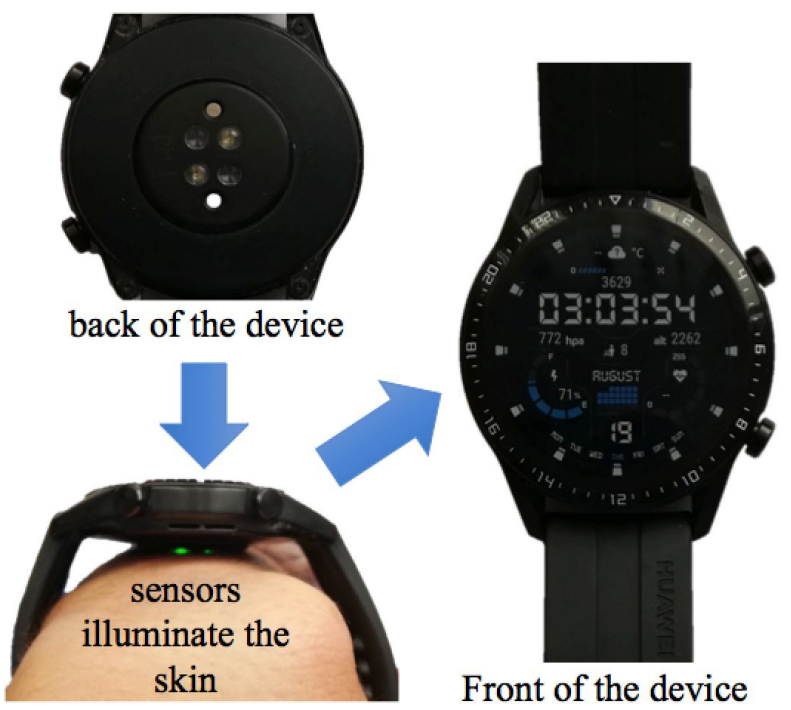

Figure 1: Smartwacth with optical sensor

For the development of the experiment, the architecture of the data logging system that is illustrated in Figure 2 was taken. First, the university professor is seen, who wears the smartwatch, which is responsible for recording stress levels 24 hours per day, except for the times while the user exercises in order to avoid errors in the estimation of stress.

These records are stored in the internal memory of the device. Second, the data is periodically downloaded in the "Health" application provided by the Huawei Company. This communication is carried out according to the protocols established by a Bluetooth communication. Finally, a screen capture is made with the Smartphone records and they are sent via WIFI to a computer where the statistical analysis of the data is carried out.

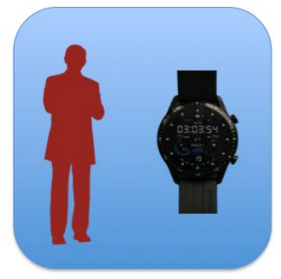

User with the smartwatch

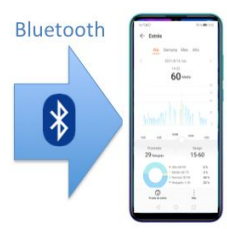

Smartphone

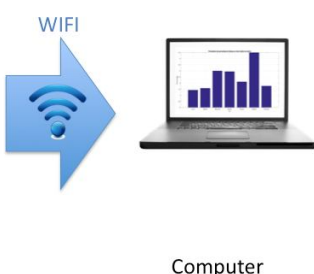

Computer
Figure 2: Data logging system architecture

For the selection of the university teacher, it was taken into account that had workloads in academic area and in the research area. The informed consent was filled out in order to guarantee that the professor could voluntarily express his intention to participate in the research. The characteristics of the experimental test are listed below:

- Type of study: case study, quantitative.

- Sample: Non-probabilistic for convenience.

- Subject to Evaluate: University Professor, 41 years old, male.

- Registration period: 24 hours a day, January 28, 2020 to January 28, 2021 (Of the 367 days, due to the electronic equipment loading processes and partial information storage, the 113-day data was discarded, for which is handled a sample of 254 days)

- Electronic Equipment: Smartwatch Huawei GT2

- Technique: Stress level estimation based on heart rate and pre-established models Subject evaluated: University Professor that works 8 hours a day from Monday to Fridays.

The information processing procedure begins with the collection of the information recorded during the period. Subsequently, the information was verified and the days where there was not a complete record of the information were discarded. This is done to avoid biases in the information.

Figure 3 shows the record of the 254 days, considered valid. This information corresponds to the daily averages of the stress records. It should be emphasized that stress measurements are taken on a scale from 0 to 100 , where 0 is the lowest value and 100 the highest. It can be seen that the significant variation of these averages where their values oscillate between 25 and 50. The general average of the entire experiment was $34.33 \%$. 


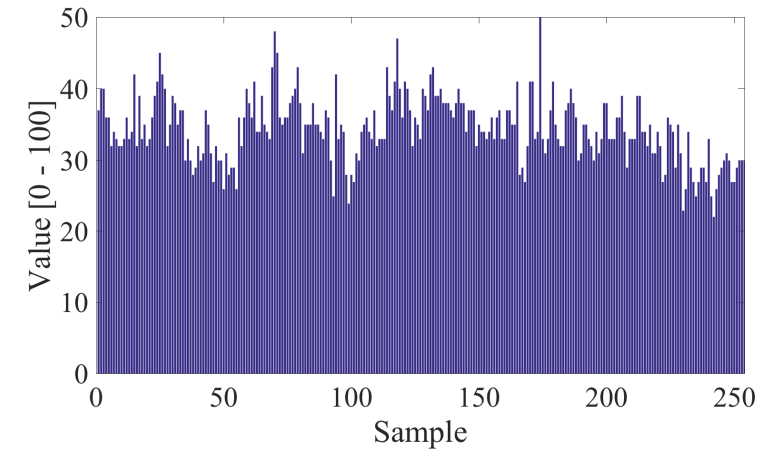

Figure 3: Daily stress averages

In addition to the daily average, records were taken of the maximum and minimum values of stress obtained by the teacher, as shown in Figure 4. The minimum values were very uniform and the majority of days coincided with the value of 15. With respect to the Maximum values of each day, ranged between 45 and 75 with a mean of $59.16 \%$.

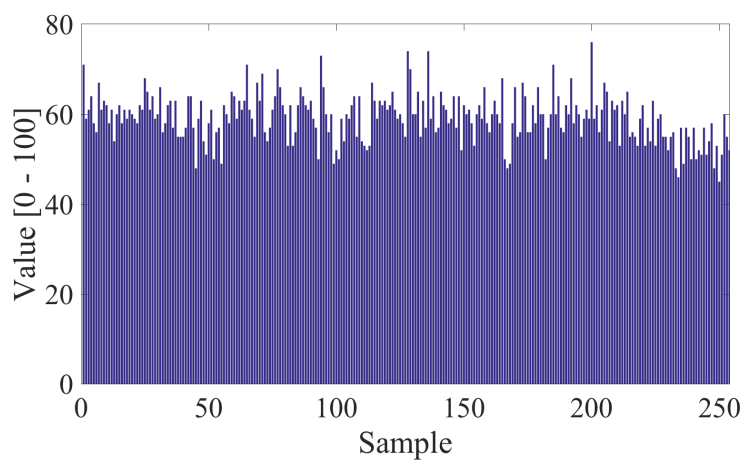

Figure 4: Maximum levels of daily stress

It should be borne in mind that the maximum stress values could arise as a result of fleeting experiences or sporadic events of the teacher. For this reason, an analysis was made of the percentage of time that the teacher remained within stress levels. For which 4 levels of stress were defined: relaxed level ( 0 to 25 ), normal level (25 to 50), medium level (50 to 75) and high level (75 to 100). These means make it possible to demonstrate in a global way, how the teacher's behavior is in terms of her emotional states. It is noteworthy that the teacher did not maintain high stress levels. In Figure 5, the percentage of time that the teacher remained at the average stress level during the day can be seen. It can be seen that these times were quite short, with some sporadic exceptions. The average value of permanence at this level was $2.48 \%$, which is quite low.

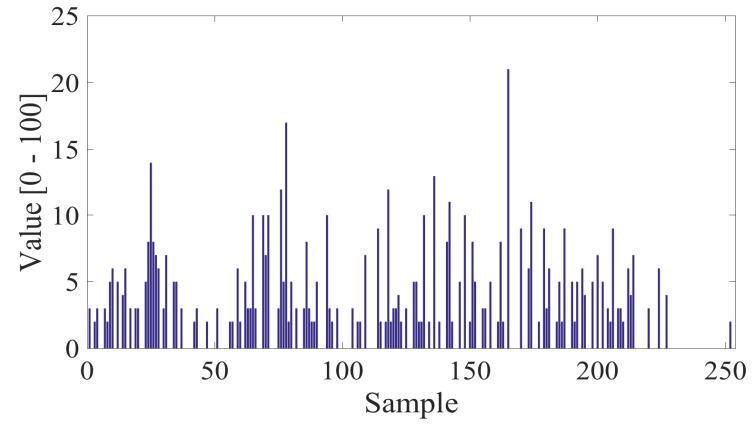

Figure 5: Percentage of time at average stress level in the day

In general terms, the teacher handled normal stress values most of the time, as can be seen in Figure 6. The fluctuations in the teacher's time at this level varied between $22 \%$ and 93\%. The mean value was $59.26 \%$.

Regarding the level of relaxed stress, the teacher had a significant average percentage (38.27\%). Figure 7 shows how this percentage changed throughout the experiment. It should be borne in mind that these measures are of a general nature. Therefore, to make a more exhaustive comparative analysis, a data grouping was carried out taking into account the days of the week. This makes it possible to more clearly identify the development of the teacher's activities and to speculate on possible improvements in the planning of tasks and the teacher's workload. This analysis is described in the results section.

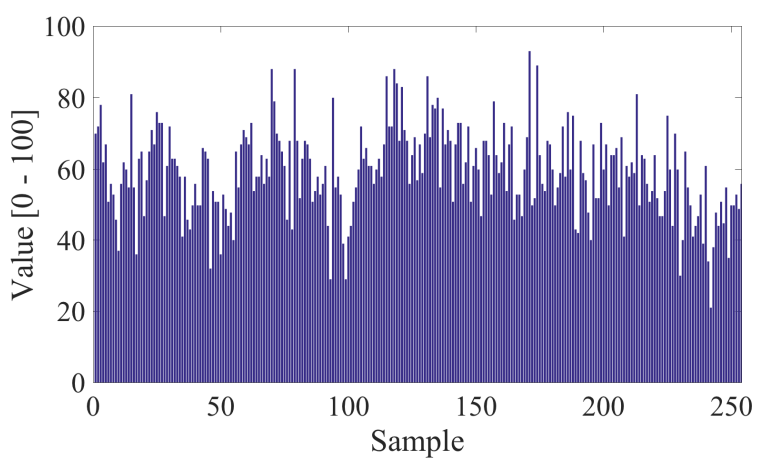

Figure 6: Percentage of time at normal stress level in the day

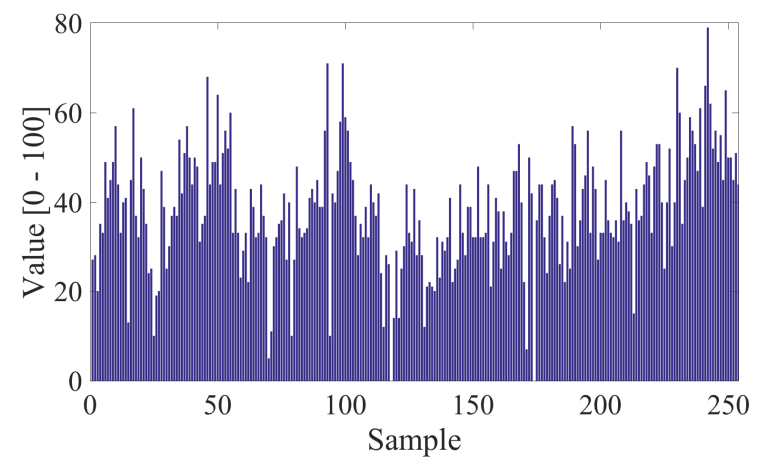

Figure 7: Time percentage at relaxed stress level in the day 
Luz Moreno et al., International Journal of Emerging Trends in Engineering Research, 10(2), February 2022, 48 - 52

\section{RESULTS}

Table 1 shows the information on stress levels according to the days of the week. It can be clearly seen that the day that the teacher handles the least average stress is Monday. The workday with the highest stress averages was Thursday with $35.41 \%$. A data that is relevant to highlight was the average of Saturday with $35.56 \%$.

In general, teachers are expected to stay relaxed on weekends, since they are days of rest, however, in this teacher evaluated, it was evident that Saturday was the day with the highest average stress. When contrasting this quantitative finding with the qualitative surveys carried out, it was found that the teacher manifested a strong extra-work load that day. The teacher said that on Saturdays, he carried out a large number of activities that ranged from shopping, taking care of children and personal projects to activities typical of the home. Otherwise it was a Sunday that was dedicated to rest.

When analyzing the average of the maximum daily stress values classified by days, it can be seen that on Wednesday there are the highest values with $59.78 \%$ (working days) and on Saturday at the weekend with $60.64 \%$. On the other hand, the lowest values occurred on Mondays and Sundays.

Table 1. Stress levels information according to the days of the week

\begin{tabular}{|c|c|c|c|c|c|c|c|}
\hline \multirow{2}{*}{ Variable } & \multicolumn{7}{|c|}{ Day of the week } \\
\cline { 2 - 9 } & $\mathbf{M}$ & $\mathbf{T u}$ & $\mathbf{W}$ & $\mathbf{T h}$ & $\mathbf{F}$ & $\mathbf{S a}$ & $\mathbf{S u}$ \\
\hline $\begin{array}{c}\text { Daily stress } \\
\text { averages }\end{array}$ & 32.51 & 34.33 & 34.43 & 35.41 & 35.03 & 35.56 & 33.03 \\
\hline $\begin{array}{c}\text { Averages of the } \\
\text { highest daily stress } \\
\text { value }\end{array}$ & 57.91 & 58.97 & 59.78 & 59.75 & 59.75 & 60.64 & 57.32 \\
\hline $\begin{array}{c}\text { Percentage } \\
\text { averages of time in } \\
\text { stress medium level }\end{array}$ & 1.43 & 1.58 & 3.00 & 2.94 & 2.11 & 4.5 & 1.76 \\
\hline $\begin{array}{c}\text { Percentage } \\
\text { averages of time in } \\
\text { stress normal level }\end{array}$ & 56.26 & 61.81 & 57.86 & 60.06 & 62.56 & 58.92 & 57.39 \\
\hline $\begin{array}{c}\text { Percentage } \\
\text { averages of time in } \\
\text { stress relaxed level }\end{array}$ & 42.31 & 36.61 & 39.14 & 37 & 35.33 & 36.58 & 40.84 \\
\hline
\end{tabular}

In the last 3 rows of Table 1 , the distribution of the percentages of time that the teacher maintained in each of the stress levels (medium, normal and relaxed) can be seen. It was evident that the day with the highest average stress level was Wednesday with 3\% and the lowest was Monday with $1.43 \%$. The highest percentage of time at the normal level was on Friday with $62.56 \%$ and the lowest on Monday with $56.6 \%$. An interesting data to analyze is the percentage of time with a relaxed stress level; in this case it is convenient to identify the maximum that corresponds to Monday with $42.31 \%$ followed by Sunday with $40.84 \%$. These values can be seen graphically in Figure 8.

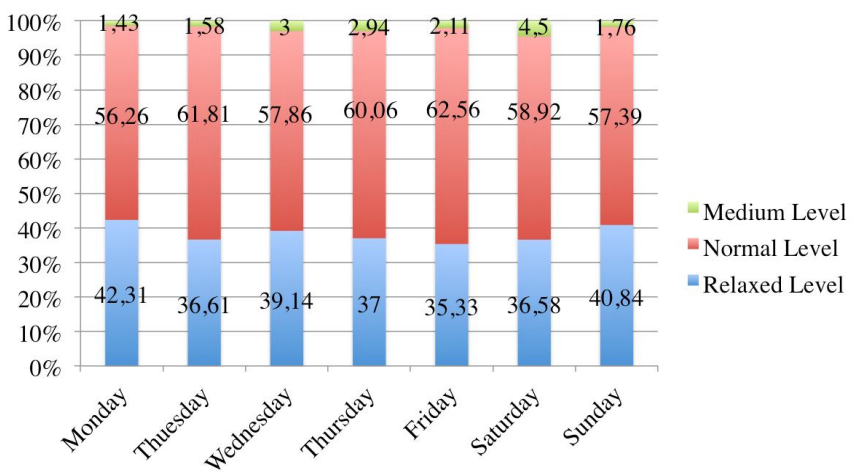

Figure 8: Distribution of the time percentage of teacher in each of the stress levels

Taking into account the results previously presented, the following suggestions could be made for this particular teacher: In case of more complex work, the teacher could preferably schedule them on Monday, Tuesday or Friday. The workload of the teacher could be rearranged by reducing the activities on Thursday.

Last but not least, it is suggested that the teacher distribute his personal activities over the two days of the weekend, since by accumulating these activities for Saturday he can suffer from a saturation of stress. It should be borne in mind that this analysis is established only for this teacher, since others may perform other functions, have a variation in time and in the amount of workload. However, this study serves as a starting point and an example to be reproduced by other teachers using the technologies described.

\section{CONCLUSION}

In the case study carried out, it was evidenced that the working day with the highest reported stress values for university teachers was Wednesday with a percentage of $59.78 \%$ and of non-working days it was Saturday with $58.92 \%$. On average, the workday with the highest stress was Thursday with $35.41 \%$ and the one with the least stress was Monday with $32.51 \%$.

It was possible to distinguish that the weekends do not necessarily correspond to a full rest, on Saturday the teacher handled the highest stress levels of the week.

It was confirmed that this technological tool that applies principles of physics to estimate heart rate and indirectly stress levels, can be useful as an analysis tool to identify the behaviour of teachers and, according to their particular characteristics, establish your workload so that you feel more comfortable in carrying out activities. 


\section{REFERENCES}

1. M. J. Mosquera, A. Kaat, M. Ring, G. Agarwal, S. Glickson, and D. Victorson, Psychometric properties of a new self-report measure of medical student stress using classic and modern test theory approaches, Health and Quality of Life Outcomes, vol. 19, no. 1, pp. $1-9,2021$.

2. S. L. McKee, X. Liu, D. M. Truong, A. C. Meinert, A. P. Daire, and S. S. Mire, The Family Adjustment Measure: Identifying Stress in Parents of Youth with Autism, Journal of Child and Family Studies, vol. 29, no. 2, pp. 592-604, 2020.

3. A. R. Kragh, F. Folke, L. Andelius, E. S. Ries, R. V. Rasmussen, and C. M. Hansen, Evaluation of tools to assess psychological distress: How to measure psychological stress reactions in citizen responders- a systematic review, BMC Emergency Medicine, vol. 19, no. 1, pp. 1-9, 2019.

4. B. A. Clough, M. Hill, M. Delaney, and L. M. Casey, Development of a measure of stigma towards occupational stress for mental health professionals, Social Psychiatry and Psychiatric Epidemiology, vol. 55, no. 7, pp. 941-951, 2020.

5. E. Yamano, A. Ishii, M. Tanaka, S. Nomura, and Y. Watanabe, Neural basis of individual differences in the response to mental stress: a magnetoencephalography study, Brain Imaging and Behavior, vol. 10, no. 4, pp. 1160-1171, 2016.

6. M. Gapen and T. Guy, Neurofeedback in the Treatment of Early Life Stress: a 'Nudge' for the Nervous System?, Current Treatment Options in Psychiatry, vol. 8, no. 3, pp. 77-94, 2021.

7. B. B. Lahey et al., Dispositional Negative Emotionality in Childhood and Adolescence Predicts Structural Variation in the Amygdala and Caudal Anterior Cingulate During Early Adulthood: Theoretically and Empirically Based Tests, Research on Child and Adolescent Psychopathology, pp. 1275-1288, 2021.

8. A. Sundaresan, B. Penchina, S. Cheong, V. Grace, A. Valero-Cabré, and A. Martel, Evaluating deep learning EEG-based mental stress classification in adolescents with autism for breathing entrainment BCI, Brain Informatics, vol. 8, no. 1, 2021.

9. Z. Hosseini, R. Delpazirian, H. Lanjanian, M. Salarifar, and P. Hassani-Abharian, Computer Gaming and Physiological Changes in the Brain: An Insight from QEEG Complexity Analysis, Applied Psychophysiology Biofeedback, vol. 46, no. 3, pp. 301-308, 2021.

10. M. Paternina, Music selector algorithm based on the mood of the user using brain-computer interface," Dep. Mechatronics Eng., University of Pamplona, Pamplona, NS, 2019.

11. V. Hoareau, C. Godin, F. Dutheil, and M. Trousselard, The Effect of Stress Management Programs on Physiological and Psychological Components of
Stress: The Influence of Baseline Physiological State, Applied Psychophysiology Biofeedback, vol. 46, no. 3, pp. 243-250, 2021.

12. T. Karthick, M. Sangeetha, M. Ramprasath, and K. Ananthajothi, Continuous Activity-Aware Stress Detection Using Sensors, Wireless Personal Communications, August, 2021.

13. V. Desai, A. Gupta, L. Andersen, B. Ronnestrand, and M. Wong, Stress-Reducing Effects of Playing a Casual Video Game among Undergraduate Students, Trends in Psychology, vol. 29, no. 3, pp. 563-579, 2021.

14. D. M. Hallman, E. M. G. Olsson, B. Von Scheéle, L. Melin, and E. Lyskov, Effects of heart rate variability biofeedback in subjects with stress-related chronic neck pain: A pilot study, Applied Psychophysiology Biofeedback, vol. 36, no. 2, pp. 71-80, 2011. 